\title{
Hepatoid adenocarcinoma of the lung metastasizing to the tonsil
}

\author{
LUCA VALLE $^{1 *}$, JOEL THOMAS $^{2 *}$, CHUL KIM $^{2}$, EVA SZABO $^{2}$, \\ G. THOMAS BROWN ${ }^{3}$, DEBORAH CITRIN ${ }^{1}$ and ARUN RAJAN ${ }^{2}$ \\ ${ }^{1}$ Radiation Oncology Branch, ${ }^{2}$ Thoracic and Gastrointestinal Oncology Branch, and ${ }^{3}$ Laboratory of Pathology, \\ National Cancer Institute, National Institutes of Health, Bethesda, MD 20892-1906, USA
}

Received September 14, 2016; Accepted January 13, 2017

DOI: $10.3892 / \mathrm{mco} .2017 .1215$

\begin{abstract}
Hepatoid adenocarcinoma (HAC) is a rare tumor that typically originates in gastrointestinal tissue, such as the stomach, but can also occasionally originate in the lung. The majority of HACs express $\alpha$-fetoprotein (AFP) on tumor cells, and serum AFP can be used as a marker of response to treatment or disease progression. HAC has a poor prognosis, although early diagnosis and aggressive treatment can result in long-term survival. To the best of our knowledge, this is the first reported case of HAC metastasizing to the tonsil. Irradiation using intensity-modulated radiation therapy (IMRT) to 30 Gray (Gy) achieved a significant, and durable tumor response. IMRT can be considered for local control of HAC at other metastatic sites of disease.
\end{abstract}

\section{Introduction}

Hepatoid adenocarcinoma (HAC) is a rare $\alpha$-fetoprotein (AFP)-producing tumor that morphologically resembles hepatocellular carcinoma. Tumor cells have eosinophilic cytoplasm, centrally located nuclei proliferating in a trabecular pattern, and express AFP, HepPar1, HEA125 and MOC31 $(1,2)$. The lung is the primary site in approximately $5 \%$ of cases of HAC (1). HAC metastasis to the brain, liver, adrenal gland, lymph nodes and bones has been previously described (1). The prognosis in patients with unresectable disease is generally poor with overall survival of 6 to 11 months (3).

In the present study, we report, to the best of our knowledge, the first case of primary lung HAC metastasizing to the tonsil.

Wide surgical excision, chemotherapy, and radiation therapy (RT) have been used to treat oropharyngeal metastases from solid tumors (4). However, the role of RT for tonsillar

Correspondence to: Dr Arun Rajan, Thoracic and Gastrointestinal Oncology Branch, National Cancer Institute, National Institutes of Health, 10 Center Drive, Bldg. 10, Room 12N226, Bethesda, MD 20892-1906, USA

E-mail: rajana@mail.nih.gov

*Contributed equally

Key words: $\alpha$-fetoprotein, tonsillar metastasis, intensity-modulated radiation therapy, palliation of symptoms, survival metastases has not been previously described. The potential benefits of palliative RT in patients with lung cancer metastasis to the tonsils include mitigation of asphyxiation risk, durable remission, and an improvement in survival (5-7). Thus, RT should be considered a potentially effective treatment for tonsillar metastasis albeit tonsillar metastases portend a poor prognosis with a high likelihood of disease at other sites (5).

\section{Case report}

A 61-year-old male presenting with left-sided chest pain due to malignant pleural effusion was diagnosed with stage IVA HAC of the lung (Fig. 1). Despite front-line chemotherapy with cisplatin and pemetrexed, he experienced multiple recurrences with metastasis to the lungs, liver, adrenal gland, and spine and received four additional lines of chemotherapy. Forty-five months after diagnosis, the patient complained of a foreign-body sensation in his throat that caused intermittent gagging and a mass was identified in the left palatine tonsillar fossa (Fig. 2A). He had no systemic complaints except intermittent fatigue and mid-back pain from a metastatic thoracic spine lesion previously treated with RT. Magnetic resonance imaging (MRI) of the neck showed asymmetry of the tonsils (left larger than right), no direct invasion of the tonsillar mass into deeper structures and no cervical lymphadenopathy (Fig. 2B). A fluorodeoxyglucose positron emission tomography (FDG-PET) scan showed a hypermetabolic left tonsillar lesion with a standard uptake value (SUV) maximum of 15.2 (Fig. 2C). Biopsy of the tonsillar mass revealed poorly differentiated carcinoma with tumor morphology resembling the previously diagnosed HAC of the lung (Fig. 2D). Immunohistochemistry results were also similar to those obtained from the diagnostic biopsy, and the tumor cells expressed cytokeratin AE1/AE3 and HepPar but not cytokeratins 5/6, p63, or AFP.

After obtaining written informed consent for participation in a protocol approved by the institutional review board at the National Cancer Institute (NCT00923065), the patient was treated with 30 Gy of intensity-modulated radiotherapy (IMRT) in 10 fractions to the left tonsillar fossa. The $2.5-\mathrm{cm}$ exophytic mass decreased to a $1.5-\mathrm{cm}$ erythematous lesion and the patient's symptoms of a foreign-body sensation and intermittent gagging resolved completely. Treatment-related mucositis and dysphagia subsided within a few weeks. Computed tomography (CT) scan of the neck performed 4 months after completion of RT showed less 

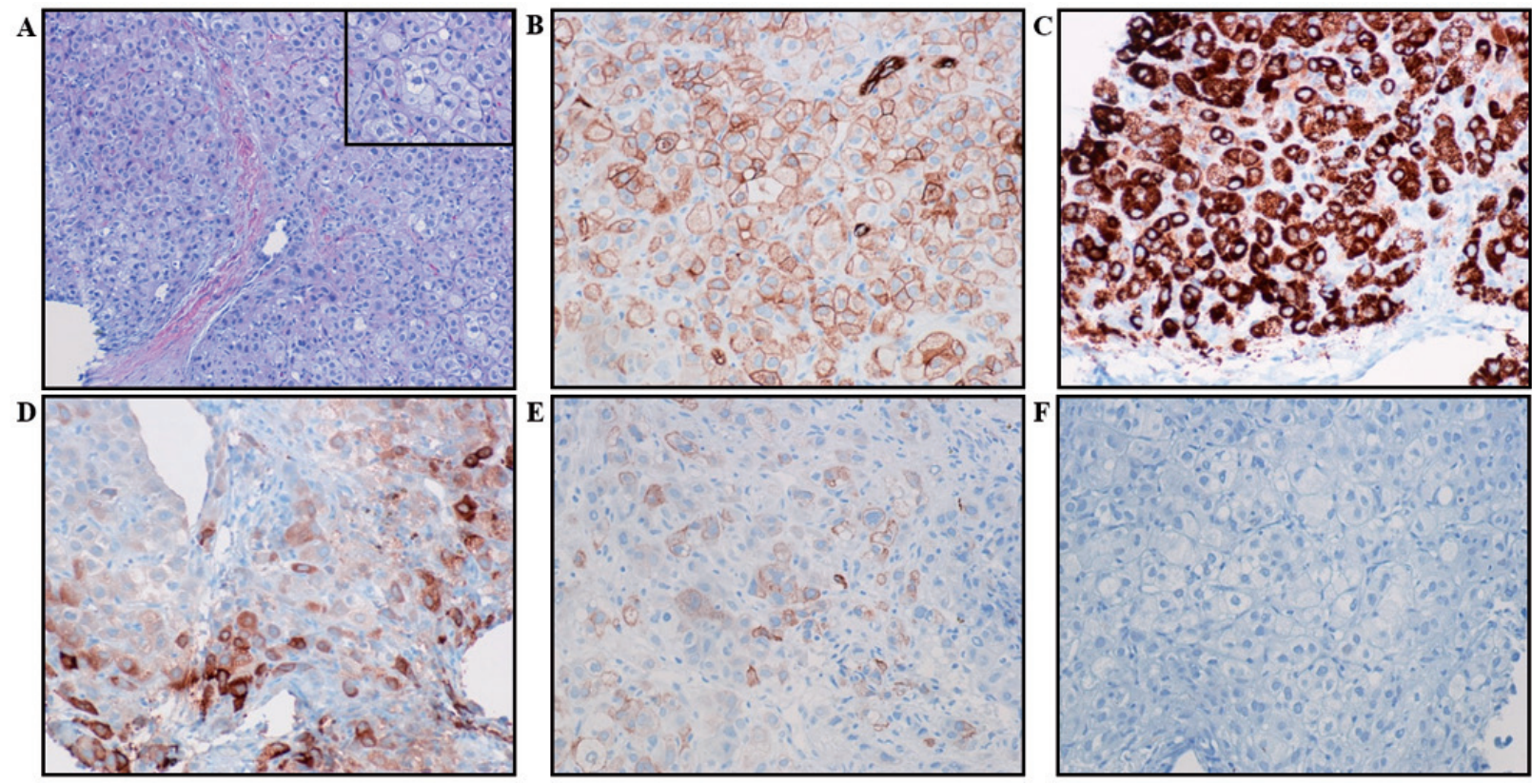

Figure. 1. Pathology of the primary tumor shows a poorly differentiated epithelioid neoplasm. Tumor cells are composed of large epithelioid cells with marked atypical nuclei with intranuclear inclusions and abundant vacuolated and eosinophilic cytoplasm (A). Immunohistochemical studies show the expression of cytokeratin AE1/AE3 (B), HepPar (C), Inhibin (moderate and patchy; D), and CK7 (weak and focal; E) and the absence of AFP (F). Tumor cells were also negative for CK20, TTF1, calretinin, WT1, CK5/6, p63, mesothelin, HBME, S-100, melanin A, monoclonal CEA, CD10, SMA, tyrosinase, HMB45, CD30, and CD31 (not shown).
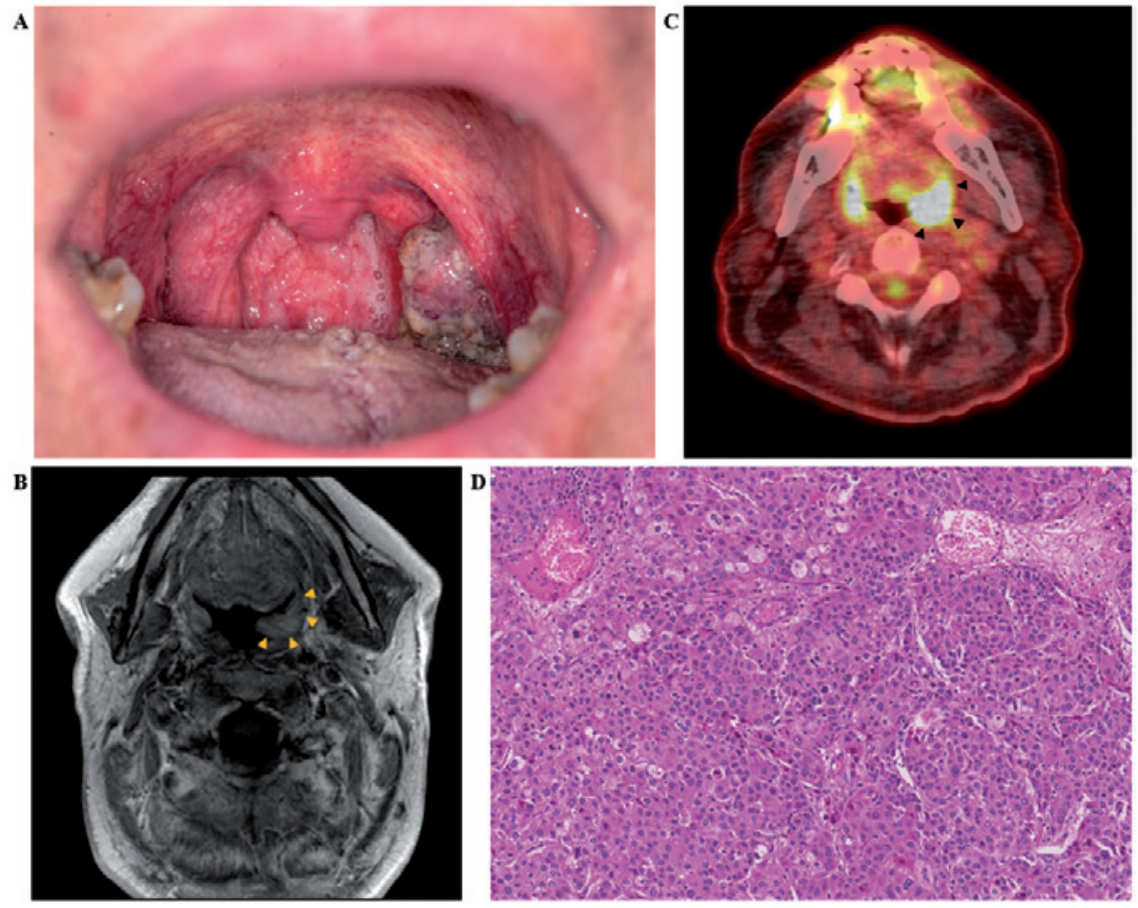

Figure. 2. A nodular mass arising in the left tonsil was identified after the patient reported a foreign body sensation in his throat (A). MRI of the neck showed enlargement of the left tonsil (indicated by arrowheads) but an absence of invasion into deeper structures (B). An FDG-PET scan demonstrated FDG uptake by the left tonsillar mass (indicated by arrowheads; C). Pathology of the left tonsil mass: tumor morphology resembles previously resected disease in the lungs (D), Immunohistochemical studies also showed a pattern consistent with the previously diagnosed HAC than with a new primary lesion (not shown).

prominence of the left tonsil compared to the pre-treatment CT and MRI of the neck. After 10 months of completion of $\mathrm{RT}$ to the tonsillar lesion the patient succumbed due to disease progression at other sites.

\section{Discussion}

The oral cavity is an infrequent site of solid tumor metastasis. In a series of 1,445 tumors of the oral cavity and oropharynx, 
$29(2 \%)$ cases were reported to be metastatic (4). Tonsillar involvement was identified in $6(0.4 \%)$ cases, with lung and liver being the most common primary sites. In another series of 1,535 tumors of the tonsil, $12(0.8 \%)$ cases were metastatic to the palatine tonsils (8). In patients with metastatic tonsillar involvement, lung cancer has been reported as the site of primary disease in 24 cases thus far, with small cell lung cancer being the dominant histology (9). Metastatic involvement of the tonsils is associated with a poor prognosis and survival following the development of tonsillar metastases is approximately 9 months, independent of the histology of the primary tumor (10).

To the best of our knowledge, this is the first report of primary lung HAC metastasizing to the tonsil. Several factors make this case unique. First, unlike the majority of HACs, this tumor did not demonstrate any immunohistochemical expression of AFP, although serum AFP was elevated and served as a reliable tumor marker. Second, despite presentation with unresectable disease, the patient was alive for 55 months after diagnosis compared to a median survival of 6-11 months. Third, the patient developed tonsillar metastasis 10 months prior to succumbing to the disease. The occurrence of metastasis in an unusual site is potentially associated with the longer natural history of the disease in this case, and required consideration of novel management strategies for palliation of symptoms related to tonsillar metastasis.

Limited data are available on the relationship between dose and response in patients with HAC treated with RT. Tsubochi and colleagues reported complete response and survival for more than 8 years in a patient with pulmonary adenocarcinoma metastatic to the tonsils and cervical lymph nodes treated with conventional RT to 50 Gy (7). Our patient had a robust response to prior palliative thoracic spine irradiation to $30 \mathrm{~Gy}$, suggesting this dose was adequate for his tumor. Thus, 30 Gy was chosen to palliate the tonsillar lesion. IMRT was employed to reduce the volume of normal tissue receiving the prescription dose and to minimize the exposure of brain and spinal cord in the event that these areas required future palliation. Our experience suggests that a lower dose of RT using modern techniques is effective in palliation of symptoms arising from tonsillar metastases from pulmonary HAC.

In conclusion, primary lung HAC metastasizing to the tonsil is a rare event that can be treated successfully with IMRT. Nevertheless, further studies are required to define the optimal dose and fractionation of RT for treatment of these uncommon cases.

\section{Acknowledgements}

The present study was supported in part by the Intramural Research Program of the NIH, National Cancer Institute, Center for Cancer Research. The study was also made possible through the NIH Medical Research Scholars Program, a public-private partnership supported jointly by the NIH and generous contributions to the Foundation for the NIH from the Doris Duke Charitable Foundation, the Howard Hughes Medical Institute, the American Association for Dental Research, the Colgate-Palmolive Company, and other private donors. For a complete list, visit the foundation website at http://www.fnih.org.

\section{References}

1. Haninger DM, Kloecker GH, Bousamra Ii M, Nowacki MR and Slone SP: Hepatoid adenocarcinoma of the lung: Report of five cases and review of the literature. Mod Pathol 27: 535-542, 2014.

2. Ishikura $\mathrm{H}$, Kanda $\mathbf{M}$, Ito $\mathbf{M}$, Nosaka $\mathrm{K}$ and Mizuno $\mathrm{K}$ : Hepatoid adenocarcinoma: A distinctive histological subtype of $\alpha$-fetoprotein-producing lung carcinoma. Virchows Arch A Pathol Anat Histopathol 417: 73-80, 1990.

3. Gavrancic T and Park YH: A novel approach using sorafenib in $\alpha$-fetoprotein-producing hepatoid adenocarcinoma of the lung. $\mathrm{J}$ Natl Compr Canc Netw 13: 387-391, quiz 391, 2015.

4. Shin SJ, Roh JL, Choi SH, Nam SY, Kim SY, Kim SB, Lee SW and Cho KJ: Metastatic carcinomas to the oral cavity and oropharynx. Korean J Pathol 46: 266-271, 2012.

5. Mastronikolis NS, Tsiropoulos GE, Chorianopoulos D, Liava AC, Stathas T and Papadas TA: Palatine tonsillar metastasis from lung adenocarcinoma. Eur Rev Med Pharmacol Sci 11: 279-282, 2007.

6. Murakawa T, Nakajima J, Fukami T, Tanaka M, Takeuchi E and Takamoto S: Tonsillar metastasis from large cell carcinoma of the lung. Jpn J Thorac Cardiovasc Surg 49: 377-380, 2001.

7. Tsubochi H, Isogami K, Sato N and Imai T: Successfully treated lingual tonsillar metastasis from bronchial adenocarcinoma. Jpn J Thorac Cardiovasc Surg 53: 455-457, 2005.

8. Hyams VJ: Differential diagnosis of neoplasia of the palatine tonsil. Clin Otolaryngol Allied Sci 3: 117-126, 1978.

9. Fukuoka K: Small cell lung cancer with isolated unitonsillar metastasis successfully treated with chemotherapy. Arch Cancer Res 4: 1-4, 2016.

10. Brownson RJ, Jaques WE, LaMonte SE and Zollinger WK: Hypernephroma metastatic to the palatine tonsils. Ann Otol Rhinol Laryngol 88: 235-240, 1979. 